\section{Update on afatinib-based combination regimens for the treatment of EGFR mutation-positive non-small-cell lung cancer}

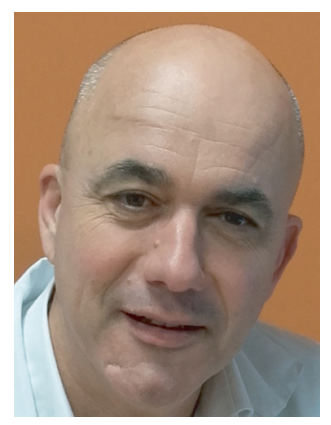

\author{
"Further head-to-head trials with afatinib as a \\ comparator are required, alongside careful analysis \\ of patient subgroups, in order to precisely define \\ which first-line EGFR-TKI will provide the best \\ outcomes in specific patient populations as well as \\ provide the most appropriate foundation for \\ subsequent sequential treatment."
}

Jaafar Bennouna ${ }^{*, 1}$

First draft submitted: 24 May 2017; Accepted for publication: 12 June 2017; Published online: 28 June 2017

Over the last decade, the first-line treatment of patients with EGFR mutation-positive non-small-cell lung cancer (NSCLC) has been revolutionized by targeted therapies. Randomized Phase III trials of the EGFR-targeting tyrosine kinase inhibitors (TKIs) gefitinib, erlotinib and afatinib have clearly shown that these agents are superior to first-line chemotherapy in terms of safety, progression-free survival (PFS) and overall response rate (ORR) [1]. As a result, all three EGFR-TKIs are considered to be current first-line standards of care for EGFR mutation-positive NSCLC.

While erlotinib and gefitinib are firstgeneration EGFR-TKIs that reversibly bind to and block EGFR signaling, second-generation afatinib has a broader and more potent spectrum of activity, irreversibly inhibiting signaling from all ErbB family receptor homodimers and heterodimers (EGFR/ErbB1, human EGFR 2/ ErbB2, ErbB3 and ErbB4) [2,3]. The recent results of LUX-Lung 7 (NCT01466660), the first randomized multicenter headto-head study to compare first-line gefitinib with afatinib, suggest that first- and second-generation EGFR-TKIs are not interchangeable, and the broad and irreversible mechanism of action of afatinib may benefit clinical outcomes in treatment-naive patients with EGFR mutationpositive NSCLC [4]. Afatinib was associated with improved efficacy across a range of clinically relevant end points compared with gefitinib in LUX-Lung 7, including median PFS (11.0 vs 10.9 months; HR: 0.73 [95\% CI: 0.57-0.95]), time to treatment failure (13.7 vs 11.5 months; HR: 0.73 [95\% CI: 0.58-0.92]) ORR (70 vs $56 \%$; odds ratio: 1.87 [95\% CI: 1.18-2.99]), while the rate of discontinuations due to treatment-related adverse events was comparable between agents [4]. Further head-to-head trials with afatinib as a comparator are required, alongside careful analysis of patient subgroups, in order to precisely define which first-line

'Department of Pneumology, Thoracic Oncology, University Hospital - Nantes, France

\section{KEYWORDS}

- afatinib • combination • EGFR

- non-small-cell lung cancer 
EGFR-TKI will provide the best outcomes in specific patient populations as well as provide the most appropriate foundation for subsequent sequential treatment.

Despite the initial clinical benefit observed with erlotinib, gefitinib and afatinib in EGFR mutation-positive NSCLC, most patients eventually experience disease progression. The development of third-generation EGFR-TKIs, such as osimertinib, has opened the door to the era of sequential EGFR-TKI therapy, at least in patients whose tumors harbor the $T 790 M$ resistance mutation (50-60\% of cases) [5]. T790Mpositive tumors are exquisitely sensitive to osimertinib, with response rates of $>60 \%[6,7]$; thus, it is now considered second-line standard-of-care in this setting.

Nevertheless, there are two key areas of unmet medical need in the EGFR mutationpositive NSCLC field. First, there is currently a paucity of efficacious second-line treatment options in approximately $50 \%$ of patients who progress after first-line EGFR-TKIs but do not harbor the T790M mutation [8]. Mechanisms of resistance to first-line EGFR-TKIs in EGFR T790M mutation-negative patients, while poorly understood, are known to be heterogeneous and involve a number of different escape pathways [8]. Consequently, there is a rationale for the development of novel treatment combination regimens that target multiple aberrant pathways in patients with T790M-negative tumors. The second area of unmet need is the development of treatment options in patients with T790Mpositive tumors following progression on osimertinib. Studies to date indicate that resistance mechanisms to third-generation TKIs are highly heterogeneous and vary from tumor to tumor. Mechanisms that have been identified include EGFR, human EGFR 2 or MET amplification, small-cell transformation and the acquisition of tertiary mutations in the EGFR gene such as $C 7975$ [5,9]. Furthermore, recent data indicate that such resistance mutations predominantly occur in cis with T790M which, in principle, will preclude the possibility of using first- or secondgeneration EGFR-TKIs following osimertinib in most cases [10]. This assertion has been substantiated in preclinical studies which show that cells resistant to third-generation EGFR-TKIs are cross-resistant to first-generation TKIs [5].

Given the heterogeneous nature of resistance mechanisms to third-generation TKIs, targeted treatment options have not been established and are an area of active investigation. One approach is to use novel combination regimens to provide broader inhibition of intracellular pathways. Several trials are ongoing, including combinations using EGFR inhibitors as a backbone [9] .

As we have previously reported [11], afatinib is a promising combination partner for anticancer therapies across a range of tumors, given its broad and irreversible inhibitory profile and low potential for drug-drug interactions [2]. Here, recent developments with afatinib-based combinations that may have particular promise in the areas of unmet need described above are discussed. Such regimens include combinations with EGFR monoclonal antibodies, such as cetuximab and nimotuzumab [12,13], IGFsignaling inhibitors, such as xentuzumab [14], chemotherapeutic agents including paclitaxel [15] and immune checkpoint inhibitors.

\section{Afatinib plus EGFR antibodies}

The notion of combining different ErbBtargeted agents such as afatinib and cetuximab to boost ErbB pathway inhibition in relapsed/ resistant EGFR mutation-positive NSCLC is supported by the promising efficacy and tolerability observed with this combination in both preclinical and early-phase clinical studies. Afatinib plus cetuximab demonstrated encouraging antitumor activity in mouse models of human NSCLC [16] as well as a Phase Ib study of heavily pretreated patients with advanced solid tumors (NCT02020577) [17]. Most relevantly, in a Phase Ib study of 126 patients with advanced EGFR mutation-positive NSCLC with acquired resistance to first-line erlotinib or gefitinib (NCT01090011), afatinib plus cetuximab conferred an ORR of $29 \%$, with similar response rates in patients harboring $T 790 M$-positive and -negative tumors (32 vs 25\%). The median PFS in patients with T790M-positive and -negative tumors was 4.8 and 4.6 months, respectively [12].

The combination of afatinib with another EGFR-directed antibody, nimotuzumab, has also shown promising activity in patients with EGFR mutation-positive NSCLC who have developed resistance to erlotinib or gefitinib. In a recent Phase Ib/II study (NCT01861223), afatinib plus nimotuzumab conferred an ORR of $23 \%$ and median PFS of 4.0 months (95\% CI: 2.3-5.7 months) [13]. In the subgroup of 27 patients categorized according to $T 790 M$ mutation status, the ORR was 18 versus 33\% and the median PFS was 3.7 versus 2.8 months 
for $T 790 M$-positive versus $T 790 M$-negative patients, respectively. An ongoing Phase I study is assessing the combination of afatinib and the EGFR monoclonal antibody necitumumab in patients with EGFR T790M mutation-negative NSCLC who have progressed on a first-generation EGFR-TKI as well as in patients with EGFR T790M mutation-positive NSCLC who have progressed on a third-generation EGFR-TKI (NCT03054038) [18].

These promising data with cetuximab and nimotuzumab remain to be confirmed by Phase III randomized studies. The mechanism of action of afatinib plus EGFR antibodies remains unknown, but appears to necessitate broad ErbB inhibition as no overall responses have been observed in trials of cetuximab in combination with erlotinib or gefitinib $[19,20]$

\section{Afatinib plus IGF-1R inhibitors}

IGF-1R signaling is known to play a critical role in the development and progression of various tumors, and has been implicated in acquired resistance to a variety of anticancer therapies, including EGFR-TKIs [21,22]. Although clinical studies of IGF-1R-targeted monotherapies have been largely disappointing to date, recent preclinical studies suggest that combination therapy with afatinib may help to overcome acquired resistance to EGFR-TKIs and thus potentially improve treatment outcomes in patients with EGFR mutation-positive NSCLC [23]. For example, combined treatment with an IGF-1R inhibitor and afatinib resulted in synergistic tumor growth inhibition in a mouse xenograft model of EGFR mutation-positive NSCLC.

An ongoing Phase Ib trial is assessing the combination of afatinib and xentuzumab (BI 836845), a humanized monoclonal antibody that binds to IGF-1/IGF-2, in patients with EGFR mutation-positive NSCLC with progression following a prior EGFR-TKI (NCT02191891) [14]. To date, this has proven to be a feasible combination which has demonstrated a manageable safety profile consisting of predominantly grade 1 and 2 adverse events commonly associated with afatinib. Encouraging activity has also been observed at the recommended Phase II dose of xentuzumab $1000 \mathrm{mg} /$ week IV + oral afatinib $40 \mathrm{mg} /$ day; 1 of the 12 patients with advanced NSCLC had a partial response and a further 10 patients had stable disease. The expansion part of the study is currently recruiting patients with NSCLC harboring sensitive EGFR mutations and acquired resistance to afatinib, in the absence of T790M mutation [14].

\section{Afatinib plus paclitaxel}

A recent randomized Phase III study, LUXLung 5 (NCT01085136), assessed the efficacy and tolerability of paclitaxel added to afatinib in heavily pretreated NSCLC patients who had progressed following $\geq 1$ line of chemotherapy, and whose tumors had progressed following initial disease control ( $\geq 12$ weeks) on erlotinib or gefitinib and subsequently on afatinib monotherapy [15]. Compared with single-agent chemotherapy alone, paclitaxel plus afatinib significantly improved PFS (median 5.6 vs 2.8 months; $\mathrm{p}=0.003)$ and ORR (32.1 vs $13.2 \% ; \mathrm{p}=0.005)$ in this late-line treatment setting, while treatment-related adverse events were consistent with those previously reported for each agent.

The investigators concluded that LUX-Lung 5 was the first prospective study to support the concept of maintaining ErbB targeting beyond formal disease progression in oncogene-addicted NSCLC, and added that the combination of afatinib plus paclitaxel warrants consideration in patients with EGFR T790M mutation-negative NSCLC who have progressed on a first-generation EGFR-TKI [15]. In contrast to LUX-Lung 5 , recent studies have demonstrated that continued exposure to erlotinib plus chemotherapy [24] or gefitinib plus chemotherapy [25] in heavily pretreated patients progressing after first-line therapy with an EGFR-TKI did not confer any additional clinical benefit, and increased toxicity versus chemotherapy alone.

\footnotetext{
Afatinib plus immune checkpoint inhibitors

The clinical success of programmed cell death 1 (PD-1) and cytotoxic T-lymphocyte-associated antigen 4 checkpoint inhibitors has generated numerous clinical studies exploring the application of these therapeutics in combinations with existing and experimental agents. Preclinical evidence suggests that activation of the oncogenic EGFR pathway enhances susceptibility of lung tumors to PD-1 blockade in mouse models [26], suggesting that the combination of PD-1 inhibitors with EGFR-TKIs may be a promising therapeutic strategy [27]. To date, however, the initial clinical studies of immunotherapies plus EGFR-TKIs have all been associated with
} 
a relatively high incidence of treatment-related toxicities, leading to study termination in several cases [27]. Afatinib plus pembrolizumab, a PD-1 inhibitor, is being assessed in an ongoing Phase I/Ib study in patients with advanced/ metastatic NSCLC with EGFR activating mutations who have progressive disease on erlotinib (NCT02364609) [28].

\section{Conclusion}

Afatinib holds promise as a therapeutic combination partner, especially in patients with limited treatment options such as those with EGFR T790M mutation-negative NSCLC that are resistant to first-line EGFR-TKIs or those with EGFR T790M mutation-positive NSCLC with acquired resistance to osimertinib.

\section{Financial \& competing interests disclosure}

$J$ Bennouna has received honoraria from Roche, Boehringer Ingelheim, BMS and AstraZeneca. The author has no other relevant affiliations or financial involvement with any organization or entity with a financial interest in or financial conflict with the subject matter or materials discussed in the manuscript apart from those disclosed.

Medical writing assistance, supported financially by Boehringer Ingelheim, was provided by L Pritchard of GeoMed, an Ashfield company, part of UDG Healthcare plc, during the preparation of this article.

\section{Open access}

This work is licensed under the AttributionNon Commercial-NoDerivatives 4.0 Unported License. To view a copy of this license, visit http://creativecommons.org/ licenses/by-nc-nd/4.0/

\section{References}

1 Russo A, Franchina T, Ricciardi GR et al. A decade of EGFR inhibition in EGFR-mutated nonsmall cell lung cancer (NSCLC): old successes and future perspectives. Oncotarget 6(29), 26814-26825 (2015).

2 Solca F, Dahl G, Zoephel A et al. Target binding properties and cellular activity of afatinib (BIBW 2992), an irreversible ErbB family blocker. J. Pharmacol. Exp. Ther. 343(2), 342-350 (2012).

3 Li D, Ambrogio L, Shimamura T et al. BIBW2992, an irreversible EGFR/HER2 inhibitor highly effective in preclinical lung cancer models. Oncogene 27(34), 4702-4711 (2008).

4 Park K, Tan EH, O’Byrne K et al. Afatinib versus gefitinib as first-line treatment of patients with EGFR mutation-positive non-small-cell lung cancer (LUX-Lung 7): a Phase IIB, open-label, randomised controlled trial. Lancet Oncol. 17, 577-589 (2016).

5 Sun JM, Park K. Can we define the optimal sequence of epidermal growth factor receptor tyrosine kinase inhibitors for the treatment of epidermal growth factor receptor-mutant nonsmall cell lung cancer? Curr. Opin. Oncol. 29(2), 89-96 (2017).

6 Janne PA, Yang JC, Kim DW et al. AZD9291 in EGFR inhibitor-resistant non-small-cell lung cancer. N. Engl. J. Med. 372(18), 1689-1699 (2015).

7 Mok TS, Wu YL, Ahn MJ et al. Osimertinib or platinum-pemetrexed in EGFR T790Mpositive lung cancer. N. Engl. J. Med. 376(7), 629-640 (2017).
8 Corallo S, D'argento E, Strippoli A et al. Treatment options for EGFR T790Mnegative EGFR tyrosine kinase inhibitorresistant non-small cell lung cancer. Target. Oncol. 12, 153-161 (2017).

9 Minari R, Bordi P, Tiseo M. Thirdgeneration epidermal growth factor receptor-tyrosine kinase inhibitors in T790M-positive non-small cell lung cancer: review on emerged mechanisms of resistance. Transl. Lung Cancer Res. 5(6), 695-708 (2016).

10 Hidaka N, Iwama E, Kubo N et al. Most T790M mutations are present on the same EGFR allele as activating mutations in patients with non-small cell lung cancer. Lung Cancer 108, 75-82 (2017).

11 Bennouna J, Moreno Vera SR. Afatinib-based combination regimens for the treatment of solid tumors: rationale, emerging strategies and recent progress. Future Oncol. 12(3), 355-372 (2016).

12 Janjigian YY, Smit EF, Groen HJ et al. Dual inhibition of EGFR with afatinib and cetuximab in kinase inhibitor-resistant EGFR-mutant lung cancer with and without T790M mutations. Cancer Discov. 4(9), 1036-1045 (2014).

13 Lee JY, Sun JM, Lim SH et al. A Phase Ib/II study of afatinib in combination with nimotuzumab in non-small cell lung cancer patients with acquired resistance to gefitinib or erlotinib. Clin. Cancer Res. 22(9), 2139-2145 (2016).

14 Park K, Cho BC, Lee KH et al. Phase Ib trial of afatinib and BI 836845 in advanced NSCLC: dose escalation and safety results. Presented at: World Conference on Lung
Cancer (WCLC). Vienna, Austria, 4-7 December 2016.

15 Schuler M, Yang JC, Park K et al. Afatinib beyond progression in patients with non-small-cell lung cancer following chemotherapy, erlotinib/gefitinib and afatinib: Phase III randomized LUX-Lung 5 trial. Ann. Oncol. 27(3), 417-423 (2016).

16 Regales L, Gong Y, Shen R et al. Dual targeting of EGFR can overcome a major drug resistance mutation in mouse models of EGFR mutant lung cancer. J. Clin. Invest. 119(10), 3000-3010 (2009).

17 Gazzah A, Boni V, Soria J-C et al. Phase Ib study of afatinib plus standard-dose cetuximab in patients with advanced solid tumours. Presented at: EORTC-NCI-AACR Molecular Targets and Cancer Therapeutics Symposium. Munich, Germany, 29 November-2 December 2016.

18 Clinical Trials database: NCT03054038. https://clinicaltrials.gov/ct2/

19 Ramalingam S, Forster J, Naret C et al. Dual inhibition of the epidermal growth factor receptor with cetuximab, an IgG1 monoclonal antibody, and gefitinib, a tyrosine kinase inhibitor, in patients with refractory non-small cell lung cancer (NSCLC): a Phase I study. J. Thorac. Oncol. 3(3), 258-264 (2008).

20 Janjigian YY, Azzoli CG, Krug LM et al. Phase I/II trial of cetuximab and erlotinib in patients with lung adenocarcinoma and acquired resistance to erlotinib. Clin. Cancer Res. 17(8), 2521-2527 (2011).

21 Denduluri SK, Idowu O, Wang Z et al. Insulin-like growth factor (IGF) signaling in tumorigenesis and the development of cancer 
drug resistance. Genes Dis. 2(1), 13-25 (2015).

22 Brahmkhatri VP, Prasanna C, Atreya HS. Insulin-like growth factor system in cancer: novel targeted therapies. Biomed. Res. Int. 2015, 538019 (2015).

23 Lee Y, Wang Y, James M, Jeong JH, You M. Inhibition of IGF1R signaling abrogates resistance to afatinib (BIBW2992) in EGFR T790M mutant lung cancer cells. Mol. Carcinog. 55(5), 991-1001 (2016).

24 Goldberg SB, Oxnard GR, Digumarthy S et al. Chemotherapy with erlotinib or chemotherapy alone in advanced non-small cell lung cancer with acquired resistance to EGFR tyrosine kinase inhibitors. Oncologist 18(11), 1214-1220 (2013).

25 Zhao NJ, Sun Z, Wang Y et al. Gefitinibintegrated regimen versus chemotherapy alone in heavily pretreated patients with epidermal growth factor receptor-mutated lung adenocarcinoma: a case-control study. Transl. Oncol. 7(4), 508-512 (2014).

26 Chen N, Fang W, Zhan J et al. Upregulation of PD-L1 by EGFR activation mediates the immune escape in EGFR-driven NSCLC: implication for optional immune targeted therapy for NSCLC patients with EGFR mutation. J. Thorac. Oncol. 10(6), 910-923 (2015).

27 Ahn MJ, Sun JM, Lee SH, Ahn JS, Park K. EGFR TKI combination with immunotherapy in non-small cell lung cancer. Expert Opin. Drug Saf. 16(4), 465-469 (2017).

28 Clinical Trials database: NCT02364609. https://clinicaltrials.gov/ct2 